\title{
Topical nitroglycerine in management of peripheral ischaemia in a neonate following arterial cannulation
}

\author{
*Medha Weerasekera ${ }^{1}$, Chamila Lakmini ${ }^{1}$, Nalaka Sanjeewa Imbulana ${ }^{1}$, Kenency Rajitha Hettiarachchi ${ }^{1}$, \\ Gayan Udara Sampath ${ }^{1}$
}

Sri Lanka Journal of Child Health, 2019; 48(3): 263-265

DOI: http://dx.doi.org/10.4038/sljch.v48i3.8765

(Key words: Accidental arterial cannulation, peripheral limb ischaemia, nitroglycerine)

\begin{abstract}
Introduction
Arterial cannulation facilitates frequent blood sampling and blood pressure monitoring in sick neonates who need intensive care. However, accidental arterial cannulation while achieving peripheral venous access is not rare $^{1}$. Ischaemia following non-heparinized intravenous fluid intended for venous infusion, eventually resulting in irreversible tissue necrosis, gangrene and loss of tissue is reported ${ }^{2}$. Preterm neonates are more susceptible to catheter related adverse effects like vasospasm, thromboembolism and peripheral ischaemia due to their small arterial diameter and inherent immaturity of haemostasis ${ }^{3}$.
\end{abstract}

Despite having variable success after removal of the cannula following heparinized saline infusion, reflex vasodilatation from warming the contralateral limb takes priority ${ }^{3}$. Initial medical management includes vasodilator therapy with topical nitroglycerine, anticoagulation with low molecular weight heparin, and thrombolysis with tissue plasminogen activator, streptokinase or urokinase ${ }^{1,3}$. However, anticoagulation and thrombolysis should be decided by weighing its risks and benefits because of the inherent susceptibility of preterm infants to intra ventricular haemorrhages ${ }^{1}$. If medical management fails, surgical options should be considered ${ }^{3,4}$. Rapidly progressive disease may require surgical thrombectomy or embolectomy ${ }^{3}$. But this may be technically difficult due to the small size of the vessels. Recent paediatric literature shows promising results on usage of topical nitroglycerine for peripheral ischaemia following arterial cannulation $^{2}$. We describe a case of successful

${ }^{1}$ Sri Jayewardenepura General Hospital, Sri Lanka *Correspondence: medhaweerasekera@gmail.com

(iD)

orcid.org/ 0000-0001-5065-1889

(Received on 15 September 2017: Accepted after revision on 24 November 2017)

The authors declare that there are no conflicts of interest

Personal funding was used for the project.

Open Access Article published under the Creative

Commons Attribution CC-BY (c) (P) License usage of topical nitroglycerine on peripheral upper limb ischaemia in a preterm neonate.

\section{Case report}

A non-asphyxiated baby boy, delivered via spontaneous vaginal delivery at 26 weeks of gestation to a 35 year old primigravida mother and weighing $880 \mathrm{~g}$, was admitted to the neonatal intensive care unit (NICU) due to prematurity and respiratory distress. His antenatal history was uncomplicated and there was no history of maternal diabetes, systemic lupus erythematosus, coagulopathy, or intra uterine growth retardation. Parents were non-consanguineous and there was no family history of thromboembolic disorders.

Baby needed ventilator support and surfactant therapy on day one. Prophylactic antibiotics were started from the time of admission. Parenteral nutrition was planned from day two as per unit protocol. Three hours after achieving peripheral access over the anterior aspect of the right distal forearm baby developed ischaemic changes on the fingertips of small, middle, ring and index fingers on day five (Figure 1).

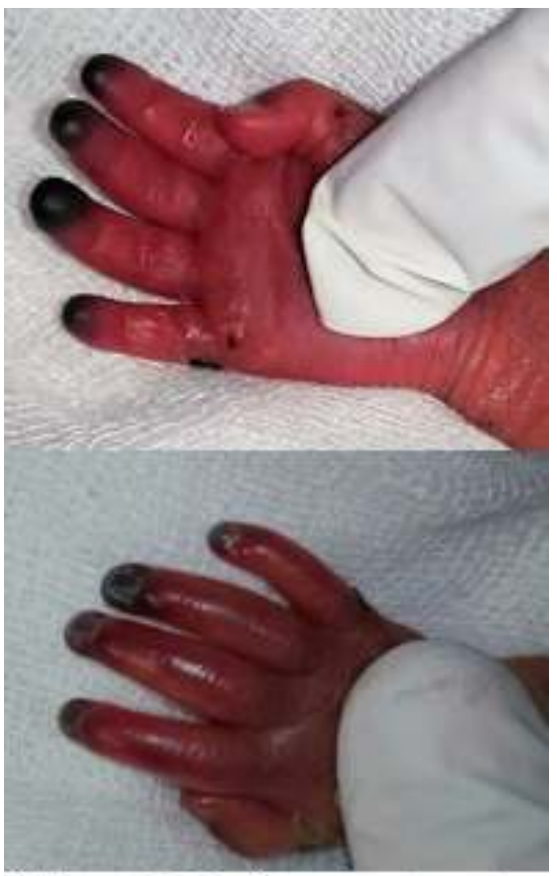

Figure 1: Before treatment 
Peripheral cannula was immediately removed and a warm dressing applied to the contralateral hand. These initial conventional measures did not show significant improvement. After liaising with surgeons, topical nitroglycerine was applied proximal to the pale line of ischaemic fingers. There was a significant improvement by reduction of oedema, peripheral vasodilatation and reduction of extent of ischaemia eighteen hours after continuous application of the therapy. Two weeks after the application of nitroglycerine, there was complete recovery and motor function of the hand was preserved. (Figure 2). Because of the obvious association with peripheral cannulation, further evaluation with thrombophilic studies was not offered.

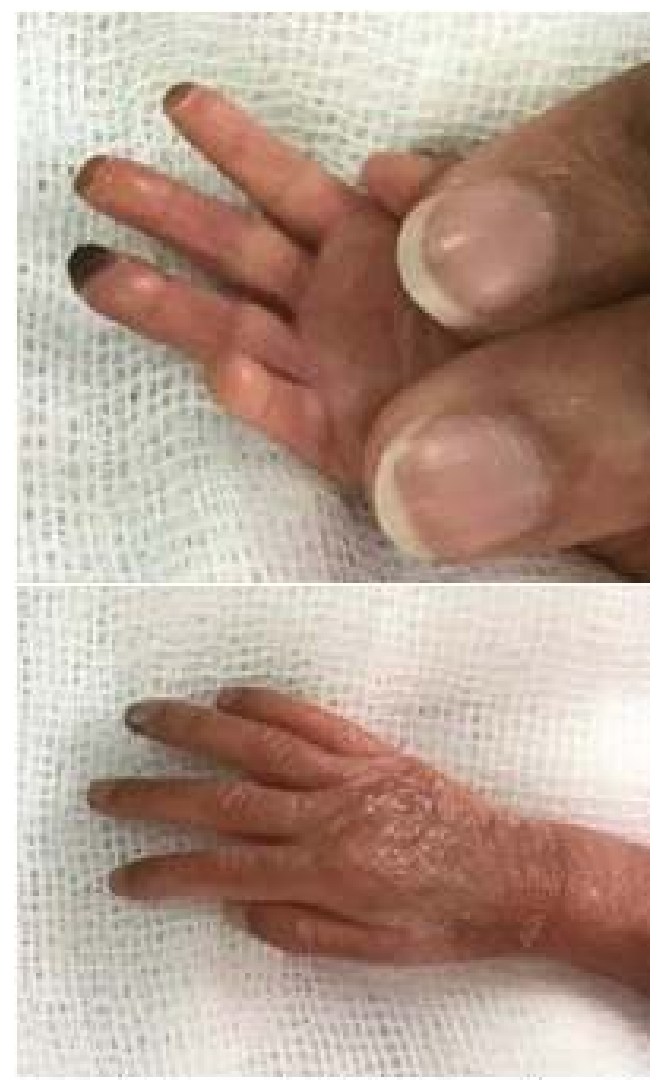

Figure 2: After treatment

\section{Discussion}

Accidental arterial cannulation is one of the well described iatrogenic morbidities in neonatal intensive care settings which may lead to distal ischaemia, necrosis and even loss of the affected $\mathrm{limb}^{2}$. Despite having a collateral circulation, there is a potential risk for development of contractures and stunting of the affected limb ${ }^{1}$. Preterm neonates have an inherent risk of developing more complications compared to a term baby because of their small calibre arteries and more immature pathways of establishing haemostasis ${ }^{3}$. Neonates achieve their maturity in coagulation at the age of 6 months ${ }^{3}$. The distal ischaemia may be secondary to reactive vasospasm, administration of hyperosmolar fluids, extravasation and thromboembolism ${ }^{2}$.

Management should take a multidisciplinary approach liaising with the vascular surgeon. An initial conservative method comprises immediate removal of the cannula, elevation of the particular limb and warming the contralateral limb in view of achieving reflex vasodilatation ${ }^{3}$. Definitive management is to restore vascular flow either medically via anticoagulation and thrombolysis or via microvascular, reconstructive surgery ${ }^{3}$. However, before anticoagulation and thrombolysis, it is mandatory to weigh the risks versus benefits as preterm neonates are more prone to get intraventricular haemorrhages. Because of this, most of the time it is reserved for definite thromboembolism. Medical management should never be an alternative in the presence of absolute indications for surgery ${ }^{3}$. Total limb ischaemia, progressive increase of gangrenous tissue and absence of blood flow in Doppler ultrasonography or angiography for more than 24 hours are considered as the absolute indications for the microvascular surgery ${ }^{3}$.

Pharmacologically, nitroglycerine is a potent vasodilator which can be absorbed through the skin in adequate therapeutic quantities ${ }^{3}$. The rate of absorption depends on the quantity and the surface area $^{3}$. Though rare, the potential side effects are tachycardia, hypotension, flushing and methaemoglobinaemia ${ }^{2,3}$. Preterm neonates are more prone to these adverse effects because their immature epidermal layer facilitates more drug absorption compared to a term baby ${ }^{3}$. Most of the adverse effects resolve spontaneously ${ }^{3}$. However, monitoring for tachycardia and hypotension throughout the treatment and measuring blood levels for methaemoglobin if the baby is having prolonged treatment are essential for early detection of side effects ${ }^{3}$. The usual dose of $2 \%$ topical nitroglycerine ointment is $4 \mathrm{~mm} / \mathrm{kg}$ which results in vasodilatation within 30 minutes and lasts for nearly 6 hours $^{2,3}$. This is equivalent to $0.2-0.5$ $\mu \mathrm{g} / \mathrm{kg} / \mathrm{min}$ dose of intravenous nitroglycerine $\mathrm{e}^{2,3,4}$. However, true occlusive disease, in contrast to vasospasm may need additional anticoagulation and thrombolysis.

Topical nitroglycerine is a successful non-invasive treatment strategy in peripheral limb ischaemia associated with arterial cannulation which leads to complete recovery and sparing of motor function of the affected $\operatorname{limb}^{3}$. Despite obvious improvement shown in case histories the research evidence is lacking on its optimal dose, timing of initiation and safety profile. 


\section{References}

1. Gault DT. Vascular compromise in newborn infants. Archives of Disease in Childhood 1992; 67:463-7.

https://doi.org/10.1136/adc.67.4_Spec_No .463

PMid: 1586194 PMCid: PMC1590504

2. Samiee-Zafarghandy S, Van den Anker JN, Ben Fadel N. Topical nitroglycerine in neonates with tissue injury: A case report and review of the literature. Paediatrics and Child Health 2014; 19:9-12.

https://doi.org/10.1093/pch/19.1.9

PMid: 24627647 PMCid: PMC3938212
3. Mosalli R, Elbaz M, Paes B. Topical nitroglycerine for neonatal arterial associated peripheral ischemia following cannulation: a case report and comprehensive literature review. Case Reports in Pediatrics 2013; 2013: Article ID 608516.

4. Arshad A, MaCarthy MJ. Management of limb ischaemia in the neonate and infant. European Journal of Vascular and Endovascular Surgery 2009; 38:61-5. https://doi.org/10.1016/j.ejvs.2009.03.010 PMid: 19362027 